\title{
Physician and Other Healthcare Personnel Responses to \\ Hospital Stroke Quality of Care Performance Feedback: A Qualitative Study
}

Joseph S. Ross, MD, $\mathrm{MHS}^{1}$; Linda S. Williams, $\mathrm{MD}^{2}$;

Teresa M. Damush, $\mathrm{PhD}^{2}$; Marianne S. Matthias, $\mathrm{PhD}^{2}$.

\author{
${ }^{1}$ Section of General Internal Medicine and the Robert Wood Johnson Foundation Clinical Scholars \\ Program, Department of Medicine, Yale University School of Medicine; Department of Health Policy and \\ Management, Yale University School of Public Health; and the Center for Outcomes Research and \\ Evaluation, Yale-New Haven Hospital System, New Haven, CT; ${ }^{2}$ VHA HSR\&D Stroke Quality \\ Enhancement Research Initiative (QUERI) and the HSR\&D Center for Health Information and \\ Communication (CHIC), Richard L. Roudebush Veterans Affairs Medical Center; and the Regenstrief \\ Institute, Indiana University School of Medicine, Indianapolis, IN
}

Corresponding author: Joseph S. Ross, MD, MHS, Section of General Internal Medicine, Yale University School of Medicine, P.O. Box 208093, New Haven, CT 06520, Phone: (203) 785-2987, Fax: (203) 7373306, E-mail: joseph.ross@yale.edu.

Word count (text only): 4265

Abstract word count: 250

References: 30

\section{Acknowledgements}

Funding/support and role of the sponsor: The project was supported by the Department of Veterans Affairs, Veterans Health Administration, Office of Performance Measurement (formerly the Office of 
Quality and Performance), and the Health Services Research and Development (HSR\&D) Service Quality Enhancement Research Initiative (QUERI) (RRP 09-184 and STR 03-168). The views expressed in this article are those of the authors and do not necessarily represent the views of the Department of Veterans Affairs. The sponsor played no role in the design of the study, analysis or interpretation of findings, or drafting the manuscript and did not review or approve the manuscript prior to submission. The authors assume full responsibility for the accuracy and completeness of the ideas presented. Dr. Ross is supported by the National Institute on Aging (K08 AG032886) and by the American Federation for Aging Research through the Paul B. Beeson Career Development Award Program. Dr. Matthias is supported through the VA HSR\&D Career Development Award Program (CDA 10-034).

Potential Conflicts of interest: Dr. Ross reports that he receives research support from the Centers of Medicare and Medicaid Services (CMS) to develop and maintain performance measures that are used for public reporting, from Medtronic, Inc. and Johnson and Johnson to develop methods of clinical trial data sharing, and from the Food and Drug Administration (FDA) to develop methods for post-market surveillance of medical devices.

Author contributions: All authors were responsible for the conception and design of this work, acquisition of data, participated in the analysis and interpretation of the data, and critically revised the manuscript for important intellectual content. Drs. Ross and Matthias drafted the manuscript and conducted the statistical analysis. Dr. Williams obtained funding. Data sharing statement: Requests for de-identified interviews (i.e., the data) which form the basis for this study can be made to the corresponding author at joseph.ross@yale.edu.

\section{ABSTRACT}

\section{Background}

Understanding how physicians and other healthcare personnel respond to hospital performance feedback initiatives may have important implications for quality improvement efforts. Our objective was 
to explore responses to the inaugural feedback of hospital performance on stroke quality of care measures among relevant physicians and personnel at U.S. Department of Veterans Health Administration (VHA) hospitals.

\section{Methods}

Qualitative interviews with hospital administrators, physicians, nurses, and quality managers at 12 VHA hospitals in the United States after the inaugural national release of the report on quality of acute stroke care processes. Interview transcripts were analyzed using an immersion/crystallization approach to identify recurrent themes.

\section{Results}

Interviews were completed with 41 individuals at 12 VHA hospitals from diverse regions of the United States; the majority were clinicians, either physicians or nurses, and nearly all had 20 years of experience or more. Interviewees described general perceptions of internal performance feedback that were both positive and negative, such as the notion that performance feedback could provide value to clinicians and hospitals, but at the same time voiced concerns about being inundated with such data. Interviewees also expressed skepticism about public reporting of performance data, citing numerous concerns and limitations. However, when interviewees described specific experiences with performance feedback, nearly all reactions were positive, including excitement, interest, and feeling validated about a job well-done.

\section{Discussion}

Physicians and other healthcare personnel described hospital performance feedback on stroke quality of care measures to be broadly valuable but identified areas of concern related to the measurement process and public reporting. 


\section{INTRODUCTION}

Measurement of quality and performance has become increasingly important in healthcare systems around the world and is nearly universal among high-income countries. In the U.S., quality measures were initially focused on process measures of care, ${ }^{1-3}$ such as the quality reporting initiatives for health plans through the National Committee for Quality Assurance's Health Plan Employer Data and Information Set. ${ }^{4}$ Over the past 5-10 years, measurement has extended to include structural and outcome measures of care and now are used for increasing numbers of physicians, hospitals, and health plans, driven in part by the U.S. Department of Health and Human Services through the Centers for Medicare \& Medicaid Services Hospital Quality Alliance initiatives on inpatient and outpatient care, as well as physician quality reporting system initiatives. ${ }^{5}$ The U.S. Department of Veterans Health Administration (VHA) has been a leader in measuring quality and performance, providing regular performance feedback to its network leaders, hospitals, and physicians to inform and improve clinical practice since the mid-1990s, with particular focus on ambulatory care quality. ${ }^{6-9}$

Surprisingly little is known about the beliefs, preferences, and experiences of hospital administrators, physicians, or clinical personnel with respect to performance feedback of clinical processes of care and outcomes. One recent nationwide survey of U.S. hospital quality directors and administrators found that public reporting of hospital quality measures exerts strong influence over local planning and improvement efforts, although concerns were expressed about the clinical meaningfulness, unintended consequences, and methods of public reporting. ${ }^{10}$ Qualitative studies have demonstrated similar beliefs and perceptions, finding that performance measure data appear to motivate and energize organizations to improve or maintain high levels of performance, despite concerns over the limitations, validity, and interpretability of publicly reported data. ${ }^{11} 12$

In February 2009, VHA decided to deliver performance feedback for the first time to all VHA hospitals on the quality of care for stroke patients, including provision of detailed performance reports. This decision offered a unique opportunity to explore clinician and administrator beliefs, preferences, 
and experiences with hospital performance feedback using qualitative methods, including views on what constitutes appropriate response to performance feedback, intended and unintended consequences, and how to ensure clinical relevance and responsiveness. Until this time, stroke care had received little attention with respect to quality and performance measures in the VHA. Understanding how physicians and other healthcare personnel respond to a new hospital performance feedback initiative may have important implications for quality improvement efforts, for stroke care specifically, as well as more generally. Our objective was to explore responses to the feedback of hospital performance on stroke quality of care measures among relevant physicians and other healthcare personnel at VHA hospitals.

\section{METHODS}

\section{Setting and Participants}

The data reported here were part of a larger project aimed at evaluating and improving the quality of stroke care in the VHA. ${ }^{13}$ Data were collected in 2010 and 2011, beginning approximately 12 months after the release of a report from VHA Office of Quality and Performance (OQP, now the Office of Performance Measurement) Stroke Special Study (hereafter referred to as the OQP Report), ${ }^{14}$ which provided VHA hospitals feedback on Joint Commission and VHA-specific stroke care quality measures. In particular, the report focused on process measures of care related to the early assessment period (i.e., dysphagia screening, use of thrombolysis), hospital care (i.e., fall risk assessment, rehabilitation consultation), and discharge care (i.e., prescription of appropriate lipid management therapies, stroke education counseling); ${ }^{14}$ the report was not risk-adjusted for hospitals' patient case-mix, since all focused on process measures of care that have specific eligibility criteria to ensure that only patients for whom the care process is appropriate are included in the measure. Facility-specific process measures were provided to an Executive Director and Director of Quality at each facility, and each facility was given time to review and make corrections to the final data. Facilities could also see aggregate data from other VHA facilities in their Veterans Integrated Service Network. 
The purpose of the current study was to evaluate the effect of reporting (the OQP report) of ischemic acute stroke care on organizational change to improve stroke quality in the VHA, as well as the perceived effect of releasing such data publicly. The current study was conducted as part of a larger intervention within the VHA to examine the impact of performance feedback on quality improvement activities related to stroke care and all interviews reported herein were conducted prior to the intervention phase of the larger study. VAMC hospitals with at least 70 ischemic stroke admissions during Federal Year 2009 and baseline performance of $<80 \%$ on two process measures of stroke care quality (dysphagia screening prior to oral intake, deep vein thrombus prophylaxis by hospital day 2) were eligible to participate. Among 153 total VAMC hospitals, 14 were eligible, 14 were invited to participate, and 12 agreed.

Among participating VAMC hospitals, no limit was placed on the number of individuals who should participate in interviews. Facilities were prompted to identify personnel who were most likely to have received the OQP report, whose clinical or administrative responsibilities included the provision of stroke care or oversight over quality initiatives, and to whom the OQP report findings were most relevant. Interviewees included neurologists, internists, Emergency Department physicians, chiefs of staff, nurses, rehabilitation staff (e.g., physical therapists and occupational therapists), and quality management staff. In total, interviews with 41 physicians and other healthcare personnel were conducted at the 12 VHA hospitals from July 2010 to December 2011. Institutional Review Board approval was received from each of the 12 VHA hospitals and interviewees provided written informed consent to participate.

\section{Interviews}

Qualitative, semi-structured interviews were conducted with VHA personnel who had engaged with the OQP Report. The interview consisted of two parts (full survey available in the Appendix), beginning after the interviewee was given a copy of the OQP report and time to review in anticipation of 
the interview. The first part focused on how personnel learned of the report, their personal responses to the report, perceived administrative and clinical responses to the report, and perceived implications of the report for improving their facility's performance scores for inpatient acute stroke care. The second part of the interview explored participants' views on public reporting of performance data in general. After the interview, additional information was asked of participants, such as demographic information, years in practice, and whether respondents had specific clinical training in stroke care. Interviews were conducted by study investigators experienced in qualitative interview methods. The majority of interviews occurred face-to-face in private rooms at each facility. Seven interviews were conducted via telephone. All interviews were audio recorded, professionally transcribed, and checked for accuracy.

\section{Data Analysis}

Data collection and analysis occurred in parallel to facilitate clarification of interview questions in subsequent interviews and to ensure that theoretical saturation (i.e., the point at which additional data become redundant, no longer leading to new theoretical insights) was reached. ${ }^{15}$ Two investigators engaged in data analysis using an immersion/crystallization approach. ${ }^{16}$ This analytic strategy is an iterative process in which analysts first immerse themselves in the data, reflect upon initial interpretations ("crystallizations"), return to the data, and reflect on and refine interpretations. ${ }^{16}$

Analysis took place in two major phases. First, analysts independently read transcripts, consciously putting aside any preconceptions, to grasp overall themes in the data. Analysts then met to discuss first impressions of the transcripts. This process continued with several transcripts until the team developed a set of working codes that represented the content of the transcripts. The analytic team continued reading transcripts, applying working codes to subsequent transcripts. Through this process, codes were modified, combined, or deleted as analysts gained a greater understanding of the data. These procedures facilitated the development of a codebook, which provided a list and description of each code, with examples from the transcripts. As the codebook was developed, data 
analysts continued to apply the consensually derived codes to subsequent transcripts, meeting weekly to discuss and refine the coding, until the codebook remained stable and consistent across transcripts.

Once the codebook was finalized, the second major phase of analysis began. In this phase, all transcripts were divided evenly between coders, who independently coded their assigned transcripts. Both team members coded every fourth transcript in common to ensure consistency in coding and maintain consensus over time. Throughout analysis, the team wrote working memos (e.g., provisional themes, questions to be further pursued) on the basis of consensus discussions and individual readings of the data, and actively searched for negative cases that would lead to alternative understandings of the data. ${ }^{1617}$ The team used NVivo software, Version 9 (QSR International, Cambridge, MA, USA) to organize transcripts and codes and facilitate easy access to coded text.

Throughout analysis, the team employed rigorous approaches to ensure validity of the data, including practicing reflexivity (i.e., continually questioning interpretations then turning to the data to verify or challenge these interpretations), striving for depth of description (i.e., seeking out the rich, particular details of participants' accounts of their experiences and opinions), and searching for alternative explanations of the data. ${ }^{18-21}$

\section{RESULTS}

Interviews were conducted with 41 individuals at 12 VHA hospitals, including 18 physicians, 17 nurses, and several others responsible for clinical care, quality, or administration. Nearly all interviewees had 20 years of clinical experience or more, although few were currently in a position where more than $50 \%$ of their time was spent engaged in clinical activities. All individuals worked at facilities that had received performance feedback on stroke quality of care from the Veterans Health Administration within the past year.

When asked to describe their perceptions of, and experience with, performance feedback and public reporting initiatives, individuals were generally skeptical. However, when asked to discuss the 
specific performance feedback received as part of the stroke project, individuals were much more complimentary and supportive. Nevertheless, individuals continued to voice concerns and experienced a range of reactions in response to receiving performance feedback.

\section{General Perceptions of Performance Feedback}

Some interviewees initially described the value, in general, of performance feedback. They identified the importance of "knowing where we [an individual facility] stand, ... important if you're trying to improve" (Stroke Care Nurse, Facility 08) and in using measurement and feedback as a means to "find out who is better" (Chief of Emergency Medicine, Facility 01), "create accountability and responsibility" (Acute Stroke Care Nurse Manager, Facility 02), and as an "opportunity for improvement." One interviewee explained:

"I have been advocating for Neurology to have some performance measures because it's the only way we are going to get on the map." (Chief of Neurology, Facility 02)

However, there was persistent concern that performance data would need to be interpreted cautiously. For instance, one interviewee raised caution that performance and outcomes will look worse if the patient population is sicker at baseline. Other interviewees pointed out that, in some situations, performance data would not be helpful, or might be misleading. For instance, one interviewee was concerned about the timeliness of the performance feedback, explaining that the more current the information, the more helpful it will be. Another interviewee reflected on the potential negative consequences of performance measurement and feedback, suggesting that performance measures could "be turned into policy that becomes a straight jacket" (Neurologist, Facility 04).

Interviewees also voiced concern about being inundated with performance data and feedback, as well as with performance measures. For instance, there was concern about the communication of performance feedback, particularly via email, leading one interviewee to explain: 
"Well you know there's a bit of a white noise problem...I probably get 200-250 emails a day." (Chief of Medicine, Facility 03)

Similarly, another interviewee reflected on this problem, saying:

"I think people...are bombarded with a lot of information every day. So getting that feedback results to the right people who can interpret and disseminate it to the right people [is important]." (Clinical Nurse Educator, Facility 09)

However, the concern was not limited to being overwhelmed with communication, but also recognized that there was a potential overabundance of measures. One interviewee, while generally favorable towards performance measurement, noted:

"At this point in time, we're so inundated with performance measures of this, that, and the other. One more, I don't think, will make a whole lot of difference." (Chief of Neurology, Facility 02)

\section{General Perceptions of Public Reporting}

Interviewees expressed even greater skepticism about public reporting of performance data. One interviewee strongly emphasized:

"Sharing [performance data] with [patients] without the opportunity first to improve things might be viewed as punitive." (Chief of Neurology, Facility 01)

Some stressed potential downsides of these initiatives, for instance, the belief that public reporting of performance data does not make a difference to patients, arguing that patients do not pick doctors and hospitals based on performance data, but based on other factors. For instance, the Chief of Medicine at one facility explained:

"[Patients] have their beliefs about their hospital, just like you have your beliefs about your doctor, and you don't care what health grade your doctor gets. If you like your doctor, you like your doctor." (Chief of Medicine, Facility 01) 
Another interviewee shared similar sentiments:

"I think [patients] are more interested in getting their care on time, not having to wait, getting the medications they need. I'm not sure they're completely engaged in this level of comparative shopping when it comes to their health care." (Neurologist, Facility 08)

Some interviewees explicitly suggested that such data should not be made publicly available because of concerns about patients' response to and ability to comprehend the performance data. One interviewee explained, "I would be very careful [with] the sort of information I give to [patients]," reasoning that sharing particular information might lead to fear, confusion, or misinterpretation (Neurologist, Facility 02). Another suggested "I don't think they'd understand everything," suggesting that perhaps only results should be reported, without specific details (Emergency Department Nurse Manager, Facility 06). And a third worried that the data would simply not be relevant to most patients, explaining:

“Let's face it, patients aren't sitting around having a stroke. You know, I mean that's different if you send out diabetes data to patients. Stroke [symptoms] are sudden and [the performance data] doesn't apply to me unless I've had a stroke." (Chief of Medicine, Facility 01)

However, not all interviewees agreed with this sentiment, as another explained:

"[Patients] are smarter than we all think." (Clinical Nurse Educator, Facility 09)

More generally, some interviewees asked rhetorically whether public reporting of performance data "is really necessary," explaining that all hospitals want to provide excellent care. One person contended that, regardless of public reporting, "We would still do the right thing" (Quality Management Director, Facility 12).

\section{Specific Experience with Performance Feedback}

When interviewees were asked to discuss the specific performance feedback received as part of the stroke project, individual reactions in response to receiving performance feedback were generally positive, including excitement and interest, although some confessed to having no memory of receiving 
this specific performance feedback. Interviewees also varied in their ratings of the importance of the performance feedback. Some described receiving feedback as a non-event, of minimal to no importance. For others, particularly those whose measured performance was worse than anticipated or who were more directly related to ongoing stroke care, receiving performance feedback stimulated, and motivated, the facility to work toward performance improvement. Such individuals described this feedback as "crucial ... because the numbers were terrible" (Acute Stroke Care Nurse Manager, Facility 02), as "very significant," leading to formation of a local committee to address the shortcomings identified in the report (Neurologist, Facility 04), and as "an impetus for change" (Chief of Neurology, Facility 05). More specifically, one participant pointed to the value of the performance feedback in calling their attention to the facility's lack of policies guiding the use of tPA, dysphagia screening, and use of NIH stroke scale in the Emergency Department:

“Without the [performance feedback] we wouldn't have known to look at that. We thought some processes were in place and they were running smoothly. And every once in a while things would happen. And we would address that, but we didn't ... We need to look at the whole system." (Rehabilitation Therapist/Polytrauma Coordinator, Facility 02)

In contrast, several participants viewed the performance feedback as validation of current quality improvement efforts related to stroke care. For instance, one Emergency Department Nurse Manager explained:

"Well, it looked like we were doing a very fine job, so I went and told my staff we were doing a very fine job (laughter). So it was very important. It's very important to show that our nurses are functioning at a high level of expertise." (Facility 06)

Similarly, a Chief of Neurology explained:

"I was very excited about this data ... I was very inspired, actually. I was also inspired that we had done so well (laughs) locally and saw it as an opportunity for, you know, if we could do that here, why couldn't they do that anywhere?" (Facility 05) 
Despite individuals' generally positive reactions to receiving performance feedback, some had concerns, about both the validity of the data and of the methods used to measure performance. For instance, one interviewee explained that a measurement of care at a single point in time is not helpful and is less relevant to understanding performance at the facility:

"A single snapshot can always be explained away." (Chief of Medicine, Facility 10)

This respondent recommended that performance should be measured over time. Another interviewee reacted with indignation when asked about their performance measurement, explaining:

"I considered it to be an invalid process; [the performance feedback] was useless because the data was recorded all wrong." (Chief of Neurology, Facility 12)

\section{DISCUSSION}

In February 2009, performance feedback was delivered for the first time to all U.S. Department of VHA hospitals on the quality of care for stroke patients, providing a unique opportunity to use qualitative methods to gain an in-depth understanding of physicians', administrators', and other personnels' beliefs, preferences, and experiences with performance feedback. Physicians and other healthcare personnel described hospital performance feedback on stroke quality of care measures to be broadly valuable but identified areas of concern related to the measurement process and public reporting. Our findings inform recent efforts to understand how hospital physicians and other healthcare personnel respond to performance feedback initiatives, as well as public reporting, and may have important implications for improving how quality improvement initiatives and performance data are communicated with hospital personnel. This is timely for VHA stroke care, since beginning in January 2015, data on Joint Commission stroke quality indicators are abstracted by external chart reviewers and reported monthly to facilities in VHA as part of routine hospital quality assessments. Moreover, with the recent U.S. Veterans Choice Act, veterans of the U.S. military who receive care at VHA hospitals have the opportunity to choose to either continue to receive care in the VHA system or instead at a non-VHA 
facility, making public reporting of quality and comparisons to the entire U.S. healthcare system critically important to inform veterans' healthcare choices.

Our study highlights the contrast between general perceptions of any quality measurement activity and actual experience. Interviewees described general perceptions of performance feedback that were both positive and negative, such as the notion that performance feedback could provide value to clinicians and hospitals, but at the same time they were concerned about being inundated with performance data and feedback. Interviewees also expressed skepticism about public reporting of performance data, citing numerous concerns and limitations. However, when interviewees described specific experiences with performance feedback, nearly all reactions were positive, including excitement, interest, the opportunity to improve, and feeling validated about a job well-done.

Our findings focused on performance feedback for stroke quality of care are consistent with two prior qualitative studies exploring hospital personnel perceptions of and reactions to measurement and reporting of hospital performance more generally. ${ }^{11}{ }^{12}$ Hafner et al. conducted focus-group interviews with 29 administrators, physicians, and nurses from 29 randomly selected Joint Commission-accredited hospitals and found that public reporting of performance measure data appears to motivate and energize organizations to improve or maintain high levels of performance. ${ }^{11}$ Interestingly, they described hospital personnel's heightened awareness of the data and intense focus on performance improvement activities, despite concerns with the limitations, validity and interpretability of publicly reported data. In 2009, Powell et al. conducted individual interviews with 59 clinical leaders and other healthcare personnel from 4 VHA hospitals on performance measurement in primary care. ${ }^{12}$ As in our study, interviewees described both positive and negative aspects of performance measurement, but the negative aspects were voiced strongly, including the concerns that performance measurement may result in inappropriate clinical care, can distract providers from patient concerns, and may have a negative effect on patient education and autonomy. ${ }^{12}$ 
Our study suggests that most clinicians, while harboring concerns about performance measurement and public reporting in general, remain responsive to and appreciative of efforts to provide performance feedback to hospitals, payers, and other quality measurement organizations. Our findings suggest that clinicians have two points of view that at times conflict, one that appreciates performance feedback and having the opportunity to better understand the quality and outcomes they were achieving in the delivery of stroke care, as well as another that worried that performance measures were not sufficiently sophisticated to account for the clinical complexity of their patients or that there were simply too many measures. These two conflicting points of view have been observed in prior research on performance feedback. For instance, in a recent national survey of hospital quality directors, ${ }^{10}$ several concerns with performance measures were described, including issues with riskadjustment, "gaming" of measures, and random variation. These concerns were echoed in the qualitative studies discussed above ${ }^{1112}$ and were voiced publicly by those describing the unintended consequences of quality measurement. ${ }^{22}{ }^{23}$ Nevertheless, these same hospital quality directors also strongly believed that performance measures could be used for important inferences about quality, accurately reflected quality of care, and that public reporting stimulated improvement. ${ }^{10}$

These findings suggest that the end-users of performance feedback should be better integrated into the quality measurement process to alleviate concerns related to the number of performance measures, as well as their utility, validity, and reliability. Hospital administrators and clinical personnel need to be engaged in the performance measurement process, and not simply be expected to respond to the performance feedback, consistent with the National Quality Forum's current Consensus Development Process. ${ }^{24}$ This may include early discussions of the focus of the performance measures, as well as the data and methodological approach, to assure that any concerns related to importance or measure validity are addressed early in the measure development process. In addition, engagement in the performance measurement process is likely influenced by the organizational cultures and leadership styles of facilities receiving feedback. Prior studies have found that fostering a sense of mutual trust, 
respect, a sense of "team," and individual accountability play a critical role in responses to performance feedback. ${ }^{25-27}$ Other studies have identified key factors related to leadership and organizational communication that distinguished high and low performing facilities, such as having a "champion" on board to emphasize the importance of performance improvement for successful change. ${ }^{28-30}$

Although our study is one of the first to examine responses to hospital performance feedback of stroke quality of care, there are several important limitations of our analysis to consider. First, we interviewed a small number of physicians from each of the 12 VHA hospitals and we focused primarily on stroke care. Our findings may not be generalizable to quality of care for other acute care conditions, or for outpatient care, although our findings are consistent with prior qualitative research studies focused on those aspects of care. ${ }^{11} 12$ Second, we only interviewed hospital personnel, including physicians, nurses, and several other administrators responsible for providing stroke care; patient perspectives on performance measurement and public reporting, and the perspectives of physicians practicing in the ambulatory setting who need to refer patients for acute stroke care, may be different. Third, interviews were focused on physicians' and other healthcare personnel's response to hospital performance, not individual performance on quality of care measures for stroke. Fourth, while our study findings inform efforts to understand hospital personnel response to performance feedback initiatives, few interviewees discussed or reflected on how performance feedback led to specific organizational changes. This may be due, in part, to the fact that we were asking interviewees to respond to a one-time quality report that was provided by VHA central office to individual facilities with no further immediate interactions or resources. One would expect that performance feedback of this type would be insufficient to stimulate organizational change at a local level. However, several interviewees did comment on specific experiences with performance feedback at their institution, identifying both ways that feedback offered local value and stimulated local concerns. Finally, the individuals who performed interviews were affiliated with the VHA's Stroke Quality Enhancement Research Initiative. This may have contributed to their ability to establish rapport with interviewees, but may also have influenced their 
interpretation of the data or created a social desirability bias, a type of response bias whereby interviewees tell interviewers what they think they want to hear. For this reason, all interviews were kept confidential in an effort to foster candid responses.

After conducting 41 in-depth interviews with physicians, nurses and other administrators responsible for providing stroke care at 12 VHA hospitals, we found that performance feedback on stroke quality of care is generally expected to be broadly valuable, but there were concerns voiced and suggestions made for improving the measurement process and public reporting. Our findings inform recent efforts to understand how hospital physicians and other healthcare personnel respond to performance feedback initiatives, as well as public reporting, and may have important implications for improving quality improvement initiatives. 


\section{REFERENCES}

1. Chassin MR, Loeb JM, Schmaltz SP, et al. Accountability measures--using measurement to promote quality improvement. N Engl J Med 2010;363(7):683-8.

2. Chassin MR, Loeb JM. The ongoing quality improvement journey: next stop, high reliability. Health Aff (Millwood) 2011;30(4):559-68.

3. Marjoua Y, Bozic KJ. Brief history of quality movement in US healthcare. Current reviews in musculoskeletal medicine 2012;5(4):265-73.

4. National Committee for Quality Assurance. HEDIS \& Quality Measurement. Secondary HEDIS \& Quality Measurement 2007. http://web.ncqa.org/tabid/59/Default.aspx.

5. U.S. Department of Health and Human Services, Centers for Medicare and Medicaid Services. Quality Measures. Secondary Quality Measures January 26, 2012. http://www.cms.gov/Medicare/Quality-Initiatives-Patient-AssessmentInstruments/QualityMeasures/index.html.

6. Demakis JG, McQueen L, Kizer KW, et al. Quality Enhancement Research Initiative (QUERI): A collaboration between research and clinical practice. Med Care 2000;38(6 Suppl 1):I17-25.

7. Doebbeling BN, Vaughn TE, Woolson RF, et al. Benchmarking Veterans Affairs Medical Centers in the delivery of preventive health services: comparison of methods. Med Care 2002;40(6):540-54.

8. Feussner JR, Kizer KW, Demakis JG. The Quality Enhancement Research Initiative (QUERI): from evidence to action. Med Care 2000;38(6 Suppl 1):I1-6.

9. Kizer KW, Demakis JG, Feussner JR. Reinventing VA health care: systematizing quality improvement and quality innovation. Med Care 2000;38(6 Suppl 1):17-16.

10. Lindenauer PK, Lagu T, Ross JS, et al. Attitudes of hospital leaders toward publicly reported measures of health care quality. JAMA internal medicine 2014;174(12):1904-11. 
11. Hafner JM, Williams SC, Koss RG, et al. The perceived impact of public reporting hospital performance data: interviews with hospital staff. International journal for quality in health care : journal of the International Society for Quality in Health Care / ISQua 2011;23(6):697-704.

12. Powell AA, White KM, Partin MR, et al. Unintended consequences of implementing a national performance measurement system into local practice. J Gen Intern Med 2012;27(4):405-12.

13. Arling G, Reeves M, Ross J, et al. Estimating and reporting on the quality of inpatient stroke care by Veterans Health Administration Medical Centers. Circ Cardiovasc Qual Outcomes 2012;5(1):4451.

14. VHA Office of Quality and Performance (OQP) Special Study. The Quality of VA Inpatient Ischemic Stroke Care, FY2007. Secondary The Quality of VA Inpatient Ischemic Stroke Care, FY2007 November 2009.

\section{http://circoutcomes.ahajournals.org/content/suppl/2011/12/06/CIRCOUTCOMES.111.961474.D}

\section{C1/HCQ200313-961474 supplemental material.pdf.}

15. Charmaz K. Constructing Grounded Theory: A Practical Guide through Qualitative Analysis. 1st ed. Thousand Oaks, CA: SAGE Publications Ltd, 2006.

16. Borkan J. Immersion/crystallization. In: Crabtree BF, Miller WL, eds. Doing Qualitative Research. Thousand Oaks, CA: SAGE Publications Ltd, 1999:179-94.

17. Agar M. The Professional Stranger. 2nd ed. San Diego, CA: Academic Press, 1996.

18. Bernard HF. Research Methods in Anthropology: Qualitative and Quantitative Approaches. Walnut Creek, CA: AltaMira Press, Inc., 2002.

19. Davies D, Dodd J. Qualitative research and the question of rigor. Qualitative Health Research 2002;12:279-89.

20. Denzin NK, Lincoln YS. Introduction: the Discipline and Practice of Qualitative Research. In: Denzin NK, Lincoln YS, eds. Strategies of Qualitative Inquiry. 2nd ed. Thousand Oaks, CA: SAGE Publications Ltd, 2003:1-45. 
21. Glaser B, Strauss A, editors. The Discovery of Grounded Theory Research: Strategies for Qualitative Research. Chicago, IL: Aldine, 1967.

22. Werner RM, Asch DA. The unintended consequences of publicly reporting quality information. JAMA 2005;293(10):1239-44.

23. Werner RM, Asch DA. Clinical concerns about clinical performance measurement. Ann Fam Med 2007;5(2):159-63.

24. National Quality Forum. Consensus Development Process. Secondary Consensus Development Process.

\section{http://www.qualityforum.org/Measuring Performance/Consensus Development Process.aspx.}

25. Myers $M$, Parchen D, Geraci $M$, et al. Using a shared governance structure to evaluate the implementation of a new model of care: the shared experience of a performance improvement committee. The Journal of nursing administration 2013;43(10):509-16.

26. Quinn GR, Le E, Soni K, et al. "Not so fast!" the complexity of attempting to decrease door-to-floor time for emergency department admissions. Joint Commission journal on quality and patient safety / Joint Commission Resources 2014;40(1):30-8.

27. Vachon B, Desorcy B, Camirand M, et al. Engaging primary care practitioners in quality improvement: making explicit the program theory of an interprofessional education intervention. BMC health services research 2013;13:106.

28. Bradley EH, Curry LA, Spatz ES, et al. Hospital strategies for reducing risk-standardized mortality rates in acute myocardial infarction. Ann Intern Med 2012;156(9):618-26.

29. Bradley EH, Curry LA, Webster TR, et al. Achieving rapid door-to-balloon times: how top hospitals improve complex clinical systems. Circulation 2006;113(8):1079-85.

30. Gabbay RA, Friedberg MW, Miller-Day M, et al. A positive deviance approach to understanding key features to improving diabetes care in the medical home. Ann Fam Med 2013;11 Suppl 1:S99107. 